\title{
La planificación anticipada
}

del cuidado en salud: alternativa

de regulación para Chile desde

el derecho comparado

y la Convención Interamericana

para la Protección de los

Derechos Humanos de las

\section{Personas Mayores*}

\section{Edison Calahorrano Latorre ${ }^{* *}$}

Resumen. La Convención Interamericana para la Protección de los Derechos Humanos de las Personas Mayores regula la obligación de los Estados parte de implementar un proceso para que la persona mayor pueda manifestar, de manera expresa, su voluntad anticipada y sus instrucciones respecto de las intervenciones en atención de la salud. Chile ha ratificado este instrumento. El presente trabajo analiza el estado de la institución desde una perspectiva comparada y considera la oportunidad de su regulación expresa en Chile.

Fecha de recepción: 4 de noviembre de 2019. Fecha de aceptación: 14 de agosto de 2020.

Para citar el artículo: CALAhorRano LatorRe, E., "La planificación anticipada del cuidado en salud: alternativa de regulación para Chile desde el derecho comparado y la Convención Interamericana para la Protección de los Derechos Humanos de las Personas Mayores", Revista de Derecho Privado, Universidad Externado de Colombia, n. ${ }^{\circ} 40$, enero-junio 2021, 201-233, Dor: https://doi. org/10.18601/01234366.n40.08.

** Universidad de Talca, Talca, Chile; candidato a doctor. Magíster en Políticas Públicas, Facultad Latinoamericana de Ciencias Sociales, Quito, Ecuador. Abogado, Pontificia Universidad Católica del Ecuador, Quito, Ecuador. Contacto: ecalahorrano@utalca.cl Orcid: 0000-0003-0408-5737. 
Palabras ClaVe: voluntades anticipadas, testamento vital, directivas anticipadas, derechos de las personas mayores, Convención Interamericana de los Derechos $\mathrm{Hu}-$ manos de las Personas Mayores.

\title{
Advance Care Planning: an Alternative of Regulation in Chile, Some Aspects from Comparative Law and the Inter American Convention on Protecting the Human Rights of Older Persons
}

\begin{abstract}
The Inter-American Convention to protect the Human Rights of Older Persons regulates the obligation of the states parties to implement a process so that the elderly person can express their advanced directives regarding health care interventions. Chile has ratified this instrument. This paper aims to analyze the state of the institution in Chile from a comparative perspective and the opportunity for its express regulation.
\end{abstract}

KeYwords: advanced directives, living will, advance care planning, elderly person rights, Inter-American Convention on the Human Rights of Older Persons.

Sumario. Introducción. I. Especificidades de la Convención Interamericana para la Protección de los Derechos Humanos de las Personas Mayores respecto a los derechos de estas personas. II. Antecedentes y evolución: desde el testamento vital hasta el modelo de planificación anticipada del cuidado en salud. III. Evolución y desarrollo en Europa. IV. Los pioneros en Latinoamérica. v. Chile y sus oportunidades. VI. La planificación anticipada del cuidado en salud en las personas adultas mayores. Conclusiones. Referencias.

\section{Introducción}

Chile está envejeciendo a un ritmo cada vez más acelerado, como lo muestra el censo $2017^{[1]}$. La población de personas mayores casi se ha duplicado en los últimos 25 años, por lo que su protección es prioritaria para la política pública.

Uno de los aspectos de mayor relevancia jurídica respecto a las personas mayores ha sido la regulación de la pérdida de la capacidad sobreviniente cuando la persona no ha dado a conocer su voluntad respecto a decisiones relacionadas con su salud.

En Estados Unidos este problema tiene una tradición de debate de más de cuarenta años, extendiéndose también a Europa, especialmente desde los años noventa, con la entrada en vigor del Convenio para la Protección de los Derechos Humanos

1 Instituto Nacional de Estadísticas, Síntesis de Resultados del Censo 2017, [en línea], 2018, 7, disponible en: https://www.censo2017.cl/descargas/home/sintesis-de-resultados-censo2017.pdf [consultado el 23 de julio de 2019]. 
y la Dignidad del Ser Humano con respecto a las Aplicaciones de la Biología y la Medicina, del 4 de abril de 1997 (Convenio de Oviedo).

En Latinoamérica las primeras legislaciones que han tratado el tema se han adoptado en los últimos diez años y el desarrollo ha sido disímil. Los textos normativos coinciden en implementar un documento jurídicamente vinculante que contenga la voluntad de la persona y sea suscrito mientras esta mantenga plena capacidad, para que sirva de guía y mandato en el momento en que el suscriptor carezca de esta.

El documento que contiene las voluntades anticipadas tiene la finalidad de garantizar la autonomía del paciente y la mejor interpretación de sus deseos y preferencias sobre salud.

La normativa más reciente se ha centrado en el proceso, más que en el instrumento, por lo que ha adquirido el nombre de planificación anticipada del cuidado en salud (advance care planning).

En Chile no existe actualmente una norma que regule esta figura, y tampoco se cuenta con normativa que agrupe las regulaciones respecto a las personas mayores, existiendo dispersión normativa en la materia.

La Ley 19.828 del 17 de diciembre de 2002 creó el Servicio Nacional del Adulto Mayor con el objetivo de mejorar la calidad de vida de esta población, mientras que la Ley 21.144 de 2019 modificó dicha norma e incorporó la cuarta edad, que parte desde los ochenta años. La Ley 20.584 de 2012, que regula los derechos del paciente, no contiene ninguna disposición respecto a planificación anticipada del cuidado en salud.

En el Diario Oficial del 7 de octubre de 2017 se publicó el texto de la Convención Interamericana para la Protección a los Derechos Humanos de las Personas Mayores (CIDHPM) $)^{2}$, la cual ${ }^{3}$ establece el derecho de la persona mayor a "manifestar su voluntad anticipada e instrucciones respecto de las intervenciones en materia de

2 En orden cronológico, Uruguay (18 de noviembre de 2016), Costa Rica (12 de diciembre de 2016), Bolivia (17 de mayo de 2017), Chile (15 de agosto de 2017), Argentina (23 de octubre de 2017). Han depositado, por su parte, su adhesión al instrumento: El Salvador (18 de abril de 2018) y Ecuador (21 de marzo de 2019).

3 Siendo su antecedente directo la Carta de San José sobre los Derechos de las Personas Mayores de América Latina y el Caribe, generada por la Comisión Económica para América Latina (CEPAL) y adoptada en la Tercera Conferencia Regional Intergubernamental sobre Envejecimiento en América Latina y el Caribe. Los documentos referidos en el preámbulo de la Convención son los Principios de las Naciones Unidas en favor de las Personas de Edad (1991); la Proclamación sobre el Envejecimiento (1992); la Declaración Política y el Plan de Acción Internacional de Madrid sobre el Envejecimiento (2002), así como instrumentos regionales tales como la Estrategia Regional de Implementación para América Latina y el Caribe del Plan de Acción Internacional de Madrid sobre el Envejecimiento (2003); la Declaración de Brasilia (2007), el Plan de Acción de la Organización Panamericana de la Salud sobre la Salud de las Personas Mayores, Incluido el Envejecimiento Activo y Saludable (2009), la Declaración de Compromiso de Puerto España (2009) y la mencionada Carta de San José sobre los Derechos de las Personas Mayores de América Latina y el Caribe (2012). 
atención de salud, incluidos los cuidados paliativos"4; a la vez que contiene el procedimiento de registro, ampliación y modificación de dicha voluntad ${ }^{5}$.

La presente investigación pretende responder la pregunta respecto de cuáles son los efectos de la ratificación de la CIDHPM y del derecho de las personas mayores a emitir voluntades anticipadas en materia sanitaria.

Con ese propósito, en primer término se verifica qué se entiende por personas mayores, a partir del desarrollo histórico y la regulación en otros ordenamientos jurídicos.

Se parte de la hipótesis de que la entrada en vigor de la CIDHPM permite a las personas mayores emitir su voluntad anticipada, por cuanto esta institución se incorporó al ordenamiento jurídico chileno, al menos con rango de ley, si bien la ausencia de legislación secundaria impediría tener claridad sobre su funcionamiento. Como alternativa para la regulación específica en Chile, se explicará el enfoque de la planificación anticipada del cuidado en salud.

El presente trabajo es de carácter dogmático, por lo que, metodológicamente, se ha recurrido al análisis de fuentes primarias como legislación, normativa reglamentaria e historia de la ley, y de fuentes secundarias, contenidas en la doctrina más actualizada. Se ha utilizado un enfoque interdisciplinario para la selección de doctrina.

Se pretende presentar una visión comparada de la institución de la planificación del cuidado en salud, por lo que se confronta la regulación de la misma en distintos ordenamientos jurídicos seleccionados por su relevancia para la evolución histórica de la institución, como para abarcar los esfuerzos reguladores en la región. Se incorpora además el análisis de jurisprudencia relevante para la comprensión de las transformaciones de la institución objeto de estudio; esta jurisprudencia corresponde al periodo de los últimos cincuenta años. Se inicia el trabajo con el análisis del contenido de la CIPDHPM haciendo énfasis en los derechos específicos incorporados y en la regulación de la voluntad anticipada; posteriormente, se revisa el origen y evolución de la figura en la legislación y la jurisprudencia comparadas; finalmente, se revisa la discusión en Chile y se explica una alternativa de regulación centrada en la planificación anticipada del cuidado en salud frente a las obligaciones contraídas con la ratificación de la CIDHPM.

4 Convención Interamericana sobre la Protección de los Derechos Humanos de las Personas Mayores, 15 de junio de 2015, art. 11 .

5 La Convención de las Naciones Unidas sobre los Derechos de las Personas con Discapacidad, que entró en vigor el 3 de mayo de 2008 y fue ratificada por Chile el 29 de julio de ese mismo año, señala en su artículo 25 literal d), sobre el consentimiento informado en el ámbito sanitario, que se "exigirán a los profesionales de la salud que presten a las personas con discapacidad atención de la misma calidad que a las demás personas sobre la base de un consentimiento libre e informado, entre otras formas mediante la sensibilización respecto de los derechos humanos, la dignidad, la autonomía y las necesidades de las personas con discapacidad a través de la capacitación y la promulgación de normas éticas para la atención de la salud en los ámbitos público y privado". A pesar de lo señalado, la norma internacional se presentaba todavía insuficiente frente al problema de la pérdida de la capacidad sobreviniente presente con frecuencia en las personas mayores. 


\section{Especificidades de la Convención Interamericana para la Protección de los Derechos Humanos de las Personas Mayores respecto a los derechos de estas personas}

La CIDHPM consolida el reconocimiento de los adultos mayores como sujetos de derechos, en una aplicación específica del enfoque de derechos humanos en materia de cuidado $^{6}$, al señalar que los Estados parte deben "facilitar la formulación y el cumplimiento de leyes y programas de prevención del abuso, abandono, negligencia, maltrato y violencia contra la persona mayor"7, así como incorporar mecanismos de protección de los derechos.

La preocupación por las personas mayores como un grupo vulnerable y la consideración de la vejez como un potencial criterio de discriminación son relativamente recientes ${ }^{8}$. La protección de las personas mayores se ha hecho necesaria ante la progresiva dificultad para el ejercicio del catálogo común de derechos por parte de este grupo. La concepción de la persona mayor como una carga por parte de los miembros de su entorno familiar la hace proclive al abandono, por lo que requiere el reforzamiento de la protección de sus derechos desde del Estado ${ }^{9}$.

Bajo el razonamiento anterior, una virtud de la Convención reside en definir de manera clara lo que significa discriminación por edad en la vejez ${ }^{10}$. De la misma manera se define el maltrato desde una perspectiva amplia, que comprende toda situación de daño directo a la vida o la integridad física; pero, además, incluye todo acto que confirme una situación de desigualdad, frecuente en el entorno intrafamiliar ${ }^{11}$. Finalmente, la Convención contiene una definición de envejecimiento activo

6 Pautassi, L., "Del boom del cuidado al ejercicio de derechos", [en línea], Sur. Revista Internacional de Derechos Humanos, vol. 13 , n. ${ }^{\circ}$ 24, 2016, 40, disponible en: https://sur.conectas.org/wp-content/ uploads/2017/02/3-sur-24-esp-laura-pautassi.pdf [consultado el 10 de julio de 2019].

7 Convención Interamericana sobre la Protección de los Derechos Humanos de las Personas Mayores, 15 de junio de 2015 , preámbulo.

8 La Observación General n. 6 del Comité de Derechos Económicos Sociales y Culturales afirma que ni en el Pacto Internacional de Derechos Civiles y Políticos ni en la Declaración Universal de Derechos Humanos se hace mención a la edad como uno de los factores de discriminación.

9 Lathrop, F., "Protección jurídica de los adultos mayores en Chile”, Revista Chilena de Derecho, vol. 36, n. ${ }^{\circ}$ 1, 2009, 84-86; Huenchuan, S. (ed.), Envejecimiento, personas mayores y Agenda 2030 para el Desarrollo Sostenible: perspectiva regional y de derechos humanos, Comisión Económica para América Latina y el Caribe (CEPAL), 2018, 91.

10 Se entiende por tal, "cualquier distinción, exclusión o restricción, basada en la edad, que tenga como objetivo o efecto anular o restringir el reconocimiento, goce o ejercicio, en igualdad de condiciones, de los derechos humanos y libertades fundamentales en la esfera política, económica, social, cultural o cualquier otra de la vida pública y privada". Convención Interamericana para la Protección de los Derechos de las Personas Mayores, 6 de junio de 2015, art. 2.

11 Lathrop, F., "Protección jurídica de los adultos mayores en Chile", cit., 85. La cidHPM asume una definición de violencia que abarca distintos tipos de abuso, incluyendo el financiero y patrimonial, maltrato físico, sexual, psicológico, explotación laboral, expulsión de su comunidad y toda forma de abandono o negligencia que tenga lugar dentro o fuera del ámbito familiar o unidad doméstica, o que sea perpetrado o tolerado por el Estado. 
y saludable, en contraposición al "viejismo"12 y a la infantilización. La garantía de la autonomía y la independencia, sin embargo, no significa excluir el derecho al cuidado ${ }^{13}$.

Las personas mayores pueden ser sujetas a múltiples formas de discriminación ${ }^{14}$, otro criterio que consta en la Convención.

El artículo 11 de la Convención regula el derecho de autodeterminación de la persona mayor respecto a la salud, mediante la referencia expresa al consentimiento informado y el deber de informar del médico. Se señala que "las instituciones públicas o privadas y los profesionales de la salud no podrán administrar ningún tratamiento, intervención o investigación de carácter médico o quirúrgico sin el consentimiento informado de la persona mayor" sujeta del mismo. Se establece también el derecho de esta a aceptar o recibir tratamientos de toda índole, así como "información clara y oportuna sobre las consecuencias y riesgos". Este derecho está comprendido en la capacidad de autodeterminación de los pacientes, pero deberá tomar en cuenta las especificidades de la persona mayor.

Finalmente se regula la obligación de los Estados parte de implementar un proceso para que la persona mayor pueda "manifestar de manera expresa su voluntad anticipada e instrucciones respecto de las intervenciones en materia de atención de la salud, incluidos los cuidados paliativos".

La Convención señala que la voluntad anticipada será recogida mediante un documento jurídicamente vinculante que puede ser revocado, ampliado o modificado en cualquier momento. Este derecho es una expresión del principio bioético de autonomía, al igual que el consentimiento informado previo a intervenciones de carácter médico; sin embargo, tiene la peculiaridad de que sus efectos inician cuando la persona ya no se encuentra en la capacidad de manifestar su voluntad ${ }^{15}$.

El artículo 12 de la Convención, por su parte, regula el derecho al cuidado a largo plazo, una de las especificidades de los derechos de las personas mayores, especialmente de la más avanzada edad, por lo que el Estado debe garantizar la preparación de los profesionales de la salud ${ }^{16}$ y de quienes ejercen el cuidado dentro

12 Dabove, M., “Autonomía y vulnerabilidad en la vejez: respuestas judiciales emblemáticas”, Revista de Derecho Privado, vol. 34, marzo, 2018, 63-67.

13 Huenchuan, S., Envejecimiento, personas mayores y Agenda 2030 para el Desarrollo Sostenible: perspectiva regional y de derechos humanos, cit., 165-182.

14 Rey, F., "La discriminación múltiple, una realidad antigua, un concepto nuevo", Revista Española de Derecho Constitucional, n. ${ }^{\circ} 84,2008,251-283$.

15 Véase Montalvo, F., "Límites a la autonomía de voluntad e instrucciones previas: un análisis desde el derecho constitucional", DS: Derecho y Salud, vol. 20, n. ${ }^{1}$, 2010, 79-82.; Boulanger, A., "Les directives anticipées et le désir de maîtrise de sa fin de vie", Médicine \& Droit, 2017, 137-139.

16 Respecto a la importancia del papel del médico de familia en el cuidado, véase LEAL, M. et al., "Papel del médico de familia en la cumplimentación y registro del documento de instrucciones previas", SEMERGEN - Medicina de Familia, vol. 41, n. ${ }^{\circ}$ 3, 2014, 166-167. 
de la familia ${ }^{17}$. Los adultos mayores, especialmente quienes sufren una vejez acompañada de patologías, incrementan progresivamente su dependencia funcional y la necesidad de recursos requeridos para el cuidado, situación que puede llevar a serias dificultades para los cuidadores familiares en sus posibilidades de asumir un regular desempeño laboral o profesional, por lo que la intervención estatal debe ser directa y eficaz, desde una perspectiva sociosanitaria.

La Convención abarca aspectos muy concretos que caracterizan los derechos de las personas mayores en otros ámbitos; sin embargo, nos hemos concentrado en los mencionados por considerarlos específicos de este grupo etario y esenciales para el entendimiento de la institución de la voluntad anticipada.

A partir de lo señalado se verifica más adelante si este instrumento constituye una meta o un punto de partida respecto al derecho de las personas mayores a emitir su voluntad anticipada en el ámbito sanitario, objetivo para el cual es necesario repasar los antecedentes y la evolución de la institución en otros ordenamientos jurídicos. A esta tarea están dedicados los siguientes títulos.

\section{Antecedentes y evolución: desde el testamento vital hasta la planificación anticipada del cuidado en salud}

Los orígenes de la institución de la voluntad anticipada se gestaron en Estados Unidos como una reacción ante el encarnizamiento terapéutico ${ }^{18}$. En 1967, la Euthanasia Society of America expuso la necesidad de contar con un documento legal que permitiera incorporar la voluntad de la persona sobre cómo deseaba ser tratada cuando no pudiera decidir por sí misma. El primer esfuerzo concreto en este sentido se debe a Louis Kutner, quien elaboró en 1969 el formato de testamento vital ${ }^{19}$.

La primera legislación se produjo en el estado de California a través de la $\mathrm{Na}$ tural Death Act. El objetivo principal fue instaurar el derecho del paciente a emitir directivas respecto a permitir o no la aplicación de tratamientos para mantener la vida en el momento en que ya no sea capaz de expresar su voluntad ${ }^{20}$. Uno de los

17 Uno de los puntos centrales en planificación anticipada del cuidado en salud se refiere a prestar atención a los cuidadores informales o del entorno familiar; al respecto, véase Troncoso, D., "Cuidado informal a mayores dependientes en Chile: quiénes cuidan y con qué políticas sociales cuentan", América Latina Hoy, vol. 71, n. ${ }^{\circ}$ 0, 2015, 86-101.

18 Se entiende por encarnizamiento terapéutico el alargamiento de la vida, por medios artificiales, sin fines curativos; para mayor detalle, véase ToRo, R., "Conocimientos y actitudes de usuarios, médicos y enfermeras sobre las instrucciones previas en el Área Asistencial Este de la Comunidad de Madrid", tesis de doctorado, Universidad de Alcalá de Henares, [en línea], Alcalá de Henares, 2014, 35 ss., disponible en: https://ebuah.uah.es/dspace/bitstream/handle/10017/20563/Tesis\%20 Rafael\%20Toro\%20Flores.pdf?sequence=1\&isAllowed=y [Consultado el 3 de julio de 2019].

19 Arbe, M., "Estudio de las instrucciones previas en el ámbito sanitario a través del ordenamiento jurídico español”, tesis de doctorado, Universidad de Salamanca, Salamanca, 2013, 33.

20 Jonsen, A., "Dying right in California. The Natural Death Act", Clinical Toxicology, vol. 13, n. ${ }^{\circ}$, 513-522. 
elementos importantes de esta legislación consistió en permitir que las voluntades puedan ser emitidas en cualquier momento de la vida de la persona; sin embargo, preveía un régimen especial para pacientes que hubieran recibido un diagnóstico terminal, con la posibilidad de renovación permanente.

\section{A. La autonomía del paciente como nuevo derecho}

La autonomía del paciente tuvo un origen propiamente jurisprudencial, mediante la sucesión de casos emblemáticos que construyeron la noción actual de la misma como derecho. El caso Quinlan, en Estados Unidos, constituye un hito fundamental para comprender los orígenes de la institución.

Karen Quinlan se encontraba en estado de coma profundo debido a un paro cardiorrespiratorio; la mantenía viva una conexión a un ventilador pulmonar y según el diagnóstico su condición era irreversible. Su padre, Joseph Quinlan, quien había sido nombrado como curador ad litem, solicitó al Tribunal Supremo de New Jersey la autorización para desconectarla del respirador, la cual fue concedida luego de concluirse que no existía posibilidad de que su estado de salud se revirtiera y que el único propósito de los cuidados era alargar artificialmente el tiempo de vida, sin que existiera un fin curativo ${ }^{21}$.

Como consecuencia del caso señalado se reguló la necesidad de designar un representante para que tome las decisiones cuando el paciente no pueda hacerlo a través de un documento denominado Durable Power of Attorney for Health Care Statute $^{22}$.

El siguiente hito se produciría con el caso de Nancy Cruzan, quien perdió su capacidad y quedó en estado vegetativo como consecuencia de un trauma craneoencefálico. La petición en este caso era terminar con la nutrición e hidratación artificial, lo cual fue admitido por la State Trial Court. Se aceptó además el testimonio de una amiga de Cruzan que señalaba el deseo de esta de no permanecer con vida en caso de llegar a encontrarse en un estado como el que en efecto había venido a aquejarla.

La Corte Suprema del estado de Missouri reversó la decisión previa. La Corte señaló que el Living Will Statute protegía la preservación de la vida, por lo que, al no existir directivas anticipadas, ni haberse designado a sus padres como representantes, estos no estaban autorizados a decidir respecto a la terminación de la alimentación e hidratación artificial como soporte vital ${ }^{23}$. La Corte de Missouri señaló que el testimonio de la amiga de Cruzan sobre su voluntad no era suficiente para interpretar

21 In Re Quinlan, 31 de marzo de 1976, Corte Suprema de New Jersey.

22 Montalvo, F., "Límites a la autonomía de voluntad e instrucciones previas: un análisis desde el derecho constitucional", cit., 77.

23 Cruzan by Cruzan v. Director, Missouri Department of Health, No. 88-1503, 25 de junio de 1990, [en línea], disponible en: https://www.law.cornell.edu/supremecourt/text/497/261 [consultado el 20 de julio de 2019]. 
la misma; y, al no existir designación expresa de sus padres como representantes, estos tampoco podían ejercer un consentimiento por representación para decidir ${ }^{24}$.

Uno de los argumentos esenciales que hizo que la Corte Suprema de Missouri se distanciara de jurisprudencia previa como la del caso Quinlan fue el carácter no terminal del estado de Nancy Cruzan. Finalmente, la Corte señaló que las pruebas presentadas respecto a las supuestas manifestaciones de voluntad de Nancy Cruzan, en conversaciones con amigos, no podían ser constatadas, por lo que no se cumplían los requisitos del testamento vital.

La Corte Suprema de Estados Unidos ratificó el fallo de la Corte de Missouri y resolvió que las personas capaces, dentro de su derecho al consentimiento informado, como parte de la autodeterminación del paciente, tienen el derecho constitucional a rechazar tratamientos de hidratación y alimentación artificial ${ }^{25}$.

En este contexto se emitió la Patient Self-Determination Act, cuya finalidad es la difusión de los documentos de directivas anticipadas entre los ciudadanos estadounidenses, especialmente a través de los centros de atención privada financiados por los sistemas Medicare y Medicaid.

La ley indicada establece expresamente el deber de informar en hospitales, enfermerías, agencias de salud del hogar y hospicios sobre el derecho de los pacientes a emitir directivas anticipadas que incluyan la voluntad respecto a permitir o rechazar tratamientos ${ }^{26}$ en caso de llegar a encontrarse privados de la capacidad para expresarla, como parte del derecho al consentimiento informado.

Otro de los hitos importantes fue la emisión del informe s.U.P.P.O.R.T. (Study to Understand Prognoses and Preferences for Outcomes and Risks of Treatments) ${ }^{27}$.

24 Ibíd.

25 La Corte Suprema de Estados Unidos señaló que el fundamento del derecho a rechazar un tratamiento es la libertad, de la cual solo podría ser privada a través del debido proceso, según la décima cuarta enmienda de la Constitución de Estados Unidos. Por lo tanto, el derecho de libertad personal permite incluso la autorización a un representante para retirar el suministro de alimentación e hidratación artificial, siempre y cuando la voluntad del paciente esté comprobada, lo cual, según la Corte Suprema de Estados Unidos, no era aplicable al caso de Nancy Cruzan. Cruzan by Cruzan v. Director, cit.

26 Las indicaciones deberán incluir la designación de un representante que pueda tomar decisiones por la persona que haya perdido la capacidad para expresar su voluntad; disposiciones escritas sobre el cuidado de su salud, incluso en relación con la donación de órganos, documentación periódica sobre la decisión de emitir directivas anticipadas, modificarlas; no denegar la atención en salud, a menos que haya sido rechazada expresamente por el paciente o exista una directiva anticipada que lo haga; que contenga una expresión clara respecto al traslado del paciente en el caso de que el médico tratante tenga objeción de conciencia sobre el cumplimiento de sus deseos; y, la implementación de campañas educativas a los profesionales de la salud sobre el uso de las directivas anticipadas. United States of America Congress, Patient Self Determination Act 1990, [en línea], 3 de abril, H.R.4449, disponible en: https://www.congress.gov/bill/101st-congress/house-bill/4449/text [consultado el 14 de julio de 2019].

27 Connors, A. et al., "A controlled trial to improve care for seriously III hospitalized patients: The Study to Understand Prognoses and Preferences for Outcomes and Risks of Treatments (SUPPORT)", JAMA, vol. 274, n. ${ }^{\circ}$ 20, 22, 1997, 1597. En la conclusión se señala: "enhancing opportunities for more patient-physician communication, although advocated as the major method for improving patient outcomes, may be inadequate to change established practices. To improve the experience of 
A partir de este informe ha venido desarrollándose el enfoque de Advance Care Planning, centrado en el proceso integral que incluye el documento de directivas anticipadas.

\section{B. Otros casos emblemáticos en el common law}

El Reino Unido tuvo un desarrollo eminentemente jurisprudencial en materia de voluntad anticipada hasta la promulgación del Mental Capacity Act en el año 2005.

El caso Bland sirvió de antecedente directo a dicha norma. Anthony Bland sufrió un grave daño en el pecho durante una estampida humana en un partido de fútbol, lo que comprometió la oxigenación de su cerebro y lo sumió en un estado vegetativo permanente. Por consenso de los médicos tratantes, se determinó que su estado era irreversible y su supervivencia dependía de ventilación, hidratación y alimentación artificiales ${ }^{28}$.

El médico tratante solicitó al juez la supresión de los procedimientos de soporte vital. Bland carecía de la capacidad para emitir su consentimiento y tampoco había suscrito voluntades anticipadas. La Cámara de los Lores señaló que no existía un deber jurídico que obligase a mantener a Bland con vida si esta dependía únicamente de los tratamientos de alimentación e hidratación artificiales; por el contrario, esto afectaría el mejor interés del paciente. El fallo concluyó además que la alimentación por tubo nasogástrico es un tratamiento médico ${ }^{29}$.

La Mental Capacity Act del año 2005 se centra en el principio del mejor interés del paciente (best interests). A partir del parágrafo 24 de esta ley se regulan las decisiones anticipadas sobre tratamiento, cuando la persona no pueda manifestar su voluntad ${ }^{30}$. La decisión anticipada puede revocarse o modificarse en cualquier momento.

La validez de la decisión anticipada depende de la claridad en la especificación del tratamiento; de la misma manera, las circunstancias en que fueron suscritas dichas decisiones no deben haber variado, y habrá un entendimiento razonable de que no hayan sucedido nuevos hechos que puedan afectar la decisión. Respecto al rechazo de tratamientos para soporte vital, la decisión anticipada operaría, únicamente, si existe una constancia expresa por escrito en la que se manifieste la voluntad de suspensión, inclusive con riesgo vital ${ }^{31}$.

seriously ill and dying patients, greater individual and societal commitment and more proactive and forceful measures may be needed".

28 Airdale NHS Trust v. Bland, 19931 ALL ER 821, 19 de noviembre de 1992, [en línea], House of Lords, disponible en: https://www.globalhealthrights.org/wp-content/uploads/2013/01/HL-1993-AiredaleNHS-Trust-v.-Bland.pdf [consultado el 25 de julio de 2019].

29 Ibíd.

30 Act of the Parliament of the United Kingdom, Mental Capacity Act 2005, chapter 9, part 1, 2005, c. 24, 14-15 [consultado el: 25 de julio de 2019].

31 Ibíd., 15. 
Otro caso emblemático reciente en el Reino Unido, esta vez resuelto por la Corte Suprema, fue el asunto Nicklinson v. The Director of Public Prosecutions ${ }^{32}$.

La Corte Suprema del Reino Unido rechazó la apelación señalando que la regulación sobre la punibilidad de quienes asistan al suicidio a una persona está justificada en la necesidad de proteger a los más débiles que puedan verse afectados por una ausencia de control en la materia ${ }^{33}$. El caso fue controvertido por cuanto el señor Nicklinson había emitido voluntades anticipadas.

Tanto el caso Nicklinson como la permanencia de la doctrina del mejor interés del paciente han sido criticados por minimizar el ejercicio de la autonomía y la autodeterminación, confundiéndolas con el interés superior clínico del médico tratante ${ }^{34}$.

En Canadá, el fundamento para consentir o rechazar un tratamiento médico se encuentra en la sección 7 del Charter of Rights and Freedom ${ }^{35}$ que protege el derecho a la vida y la libertad, así como a la igualdad en el goce de derechos.

La regulación sobre la materia tiene un origen jurisprudencial y se desarrolla especialmente a nivel provincial ${ }^{36}$. Desde la norma Bill C-14${ }^{[37]}$, como consecuen-

32 The Supreme Court of United Kingdom, R. Nicklinson and another v. The Director of Public Prosecution, [en línea], uKsc 38, 25 de junio de 2014, disponible en: https://www.supremecourt.uk/cases/ uksc-2013-0235.html [consultado el 20 de julio de 2019].

33 Ibíd., c. 111-118.

34 Sobre las críticas e intenciones de reforma del criterio del mejor interés del paciente contenido en la Mental Capacity Act véase TAYLOR, J., "What are 'best interests'? A critical evaluation of 'best interests' decision-making in clinical practice”, Medical Law Review, vol. 24., n. ${ }^{\circ} 2 ., 2016,196-205$; Heywood, R., "Revisiting advance decision making under the Mental Capacity Act 2005: a tale of mixed messages", Medical Law Review, vol. 23, n. ${ }^{\circ}$ 1, 92-100.

35 Act of the Parliament of the United Kingdom, Constitution Act 1982, 17 de abril.

36 Jurisprudencialmente han aportado a la configuración de la obligatoriedad del contenido de las directivas anticipadas los casos de la Corte de Apelaciones de la provincia de Ontario Malette v. Shulman et al. 72 O.R., 2. a ed., 417, de 30 de marzo de 1990, y Fleming v. Reid, de 28 de junio de 1991, que establecieron la obligación del médico de respetar el contenido de las directivas anticipadas sobre el rechazo de tratamientos, inclusive si ese rechazo puede implicar la muerte. Estos esfuerzos jurisprudenciales cimentaron las bases para las primeras leyes provinciales, como la Health Care Consent Act de 1996, de la provincia de Ontario, y la Health Care (Consent) and Care (Facillity Admission) Act de 1996, de Columbia Británica.

37 Esta normativa entró en vigor el 6 de junio de 2016 y modifica el Código Criminal de Canadá eximiendo de responsabilidad a quien auxilie a una persona al suicidio o le administre sustancias nocivas con el fin de que los profesionales médicos puedan practicar el suicidio asistido. Se establecen además las salvaguardas y criterios de elegibilidad para la práctica del suicidio asistido; y se regula la obligación de los profesionales médicos y farmacéuticos que reciban requerimientos de asistencia al suicidio de informar el desarrollo del procedimiento.

Los criterios de elegibilidad son señalados en el apartado 241.2(1), y en virtud de ellos el paciente debe provenir del sistema de asistencia de salud del Gobierno de Canadá, debe tener 18 años y ser capaz de decidir respecto a su salud, tener una condición médica grave e irreversible, realizar un requerimiento de asistencia médica en la muerte, que no haya sido forzado, y emitir consentimiento informado respecto a la asistencia médica para la muerte que incluya la entrega de información sobre la posibilidad de recibir tratamientos para el dolor y cuidados paliativos.

Las salvaguardas reguladas en el apartado 241.2 (3) prevén que la persona consienta la asistencia médica para la muerte por escrito y ante dos testigos. 
cia de la discusión sobre el suicidio asistido en el caso Carter $^{38}$, es derecho de los ciudadanos canadienses mayores de edad solicitar asistencia médica para practicar el suicidio asistido ${ }^{39}$ si se encuentran afectados por una enfermedad que los someta a sufrimiento intolerable.

La implementación de Advance Care Planning se ha consolidado en Canadá, a través de la política pública del National Framework que incentiva el uso y divulgación del derecho a la planificación anticipada del cuidado en salud. La concentración en la población de personas mayores es una de las claves de esta política pública, especialmente en la planificación en el momento de plena voluntad, a través de la atención primaria y geriátrica ${ }^{40}$.

\section{Evolución y desarrollo en Europa}

Algunos países de Europa desarrollaron legislación referente a directivas anticipadas tempranamente ${ }^{41}$; sin embargo, el Convenio para la Protección de los Derechos Humanos y la Dignidad del Ser Humano con respecto a las Aplicaciones de la Biología y la Medicina, del 4 de abril de 1997 (Convenio de Oviedo), es la base de la legislación sobre directivas anticipadas en la Unión Europea.

El Convenio de Oviedo introduce en su artículo 5 el derecho del paciente a que no se realice ninguna intervención médica sin su consentimiento informado. Regula directamente las directivas anticipadas como "deseos expresados anteriormente", y en su artículo 9 señala que "serán tomados en consideración los deseos expresados anteriormente con respecto a una intervención médica por un paciente que, en el momento de la misma, no se encuentre en situación de expresar su voluntad".

Para mayor referencia véase el texto de Bill C-14, [en línea], 17 de junio de 2016, disponible en: http://laws-lois.justice.gc.ca/PDF/2016_3.pdf

38 Para una revisión crítica que acusa al texto de Bill C-14 como reducido frente a la amplitud de la sentencia en el caso Carter v. Canadá, véase Macfarlane, E., "Dialogue, remedies and positive rights: Carter v. Canada as a Microcosm of past and future issues under the Charter of Rights and Freedom", Ottawa Law Review, vol. 49, n. 1, 2017, 107-129.

39 The Supreme Court of Canada, Carter v. Canadá (Attorney General), [en línea], 2015 sCc 5, [2015] 1 s.C.R. 331 , de 6 de febrero de 2015, disponible en: https://scc-csc.lexum.com/scc-csc/scc-csc/en/ item/14637/index.do [consultado el 10 de julio de 2019].

40 Canadian Hospice Palliative Care Association, Advance Care Planning in Canada. National Framework, [en línea], 2012, 7, 23, 1-29, disponible en: http://www.advancecareplanning.ca/wp-content/uploads/2016/08/ACP-Framework-2012-ENG.pdf [consultado el 18 de julio de 2019].

41 Es el caso de la Ley de Tutela en la Asistencia (Betreuungsgestz) del 1. ${ }^{\circ}$ de enero de 1992, en Alemania, que regulaba el nombramiento de un representante para los pacientes cuando no pudieran señalar su voluntad. De la misma manera puede verificarse en las leyes danesas 351 del 14 de mayo de 1992 y 482 del 1. ${ }^{\circ}$ de julio de 1998, que regulan el testamento vital y el derecho a rechazar tratamientos médicos. En Francia se inició el debate sobre el tema con las leyes bioéticas de 1994. 
El Tribunal Europeo de Derechos Humanos ha desarrollado jurisprudencia importante sobre suicidio asistido, como la referida a los casos Pretty v. Reino Unido, Haas v. Suiza ${ }^{42}$ y Gross v. Suiza ${ }^{43}$.

Respecto a voluntades anticipadas, un caso trascendental sometido al tribunal regional fue Lambert v. Francia. A Vincent Lambert le fue diagnosticado un estado vegetativo permanente. Una vez que la opinión de los médicos tratantes fue unánime respecto a la irreversibilidad de su estado, la esposa de Lambert solicitó a la justicia francesa la autorización para detener la alimentación e hidratación artificial que lo mantenía con vida, señalando que esa habría sido la voluntad del paciente indicada en conversaciones mantenidas sobre el tema. Lambert no había suscrito documento de voluntades anticipadas. El 24 de junio de 2014 el Consejo de Estado respaldó la decisión de retirarle los medios de soporte vital, después de haber solicitado el peritaje de tres médicos designados por el organismo. Los padres de Lambert y dos hermanos interpusieron una demanda ante el Tribunal Europeo de Derechos Humanos para evitar el cumplimiento del fallo, argumentando como fundamento la violación al derecho a la vida, a no recibir torturas y a la integridad, contenidos en los artículos 2, 3 y 8 del Convenio Europeo de Derechos Humanos ${ }^{44}$.

El Tribunal Europeo señaló que la ley francesa sobre los derechos de los pacientes y del final de la vida era imprecisa respecto a definir cuál es el estado terminal de la persona y cuáles los requisitos para el rechazo a las medidas de nutrición e hidratación artificial con el fin de evitar el encarnizamiento terapéutico ${ }^{45}$, pero concluyó que el Consejo de Estado francés, en su resolución, no violó el artículo 2 del Convenio Europeo de Derechos Humanos, por cuanto cumplió con sus obligaciones positivas para recabar la voluntad de Lambert.

El Convenio de Oviedo y la jurisprudencia regional han influido en el surgimiento y las modificaciones de la normativa nacional de los países europeos sobre la materia. En el siguiente apartado se consideran los hitos más importantes.

\section{A. Las voluntades anticipadas en legislaciones nacionales}

La salud y los derechos de la persona al final de la vida han sido preocupación prioritaria de la legislación en Francia ${ }^{46}$. Es así que la Ley 2002-303 regula los derechos del paciente, entre ellos, el consentimiento informado y el derecho a la información

42 Tribunal Europeo de Derechos Humanos, Haas v. Suiza, A 31322/07; 27 de junio de 2011, c. 57-61.

43 Tribunal Europeo de Derechos Humanos, Gross v. Suiza, A 67810/10, 30 de septiembre de 2014, c. 32-37.

44 Tribunal Europeo de Derechos Humanos, Lambert v. Francia, 46043/14, 5 de junio de 2015, c. 87 88.

45 Tribunal Europeo de Derechos Humanos, Lambert vs. Francia, 46043/14, 5 de junio de 2015, c. 149-182.

46 Nicolas-Vullierme, L. et al., "De l'opportunité d'une nouvelle loi sur la fin de vie?", Médecine \& Droit, vol. 2018, n. ${ }^{\circ}$ 152, 1, 109. 
sobre los tratamientos médicos que vayan a aplicársele. Esta ley prevé la posibilidad de nombrar un representante que decida cuando el paciente ya no esté en la capacidad de hacerlo ${ }^{47}$. A partir del dramático caso de Vincent Humbert ${ }^{48}$ se promulgó la Ley 2005-370 del 22 de abril de 2005 o Ley Leonetti con el objeto de regular expresamente las directivas anticipadas.

El artículo 7 de la ley mencionada insertó en el Código de Salud el artículo L 1111-11 que señalaba que cualquier persona mayor de edad podía redactar directivas anticipadas para el caso de que en un futuro no pudiera expresar sus deseos; directivas que podían ser revocadas en cualquier momento ${ }^{49}$.

El caso Lambert motivó la promulgación de la Ley 2016-87 del 2 de febrero de 2016. El artículo 2, que inserta el artículo L 1110-1-5 en el Código de la Salud, define a la alimentación e hidratación como un tratamiento médico, por lo que puede ser rechazado con consentimiento del paciente o su representante, si su única finalidad es el alargamiento artificial de la vida ${ }^{50}$. El artículo 3 introduce en el Código de la Salud el artículo L 1110-5-2, por el cual, a petición del paciente, se puede administrar una sedación profunda ${ }^{51}$ y continua que cause alteración de la conciencia hasta conducir a la muerte ${ }^{52}$.

Respecto a las directivas anticipadas, el artículo 8 de la Ley 2016-87 señala que cualquier persona mayor de edad puede redactarlas. Las directivas anticipadas pueden expresar la voluntad de la persona con respecto al final de su vida, incluyendo las condiciones de limitación, finalización o rechazo del tratamiento o actos médicos. En todo momento las directivas son revisables y revocables.

Las directivas anticipadas son vinculantes para el médico en cualquier investigación, intervención o decisión de tratamiento, excepto en el caso de una emergencia que ponga en peligro la vida o cuando parezcan manifiestamente inapropiadas.

La decisión de negarse a aplicar directivas anticipadas, juzgadas por el médico como manifiestamente inapropiadas o en desacuerdo con la situación médica del paciente, se toma después de un procedimiento colegiado y se registra en el expediente médico.

Las directivas anticipadas se archivan en un registro nacional que está sujeto a un procesamiento automático. Cuando se han incorporado a este registro, el mismo envía regularmente un recordatorio de su existencia a su autor.

47 Parlamento Francés, Ley 2002-303 del 4 de marzo de 2002, art. L1110-4.

48 Como referencia al caso, véase PEREIRA, R., "Human handicap and self-determination: compassion and insensibility in the Vincent Humbert case", en História, Ciências, Saúde-Manguinhos, vol. 14, n. ${ }^{\circ} 1,2007,119-134$.

49 Parlamento Francés, Ley 2005-370 del 22 de abril de 2005, art. 7.

50 Parlamento Francés, Ley 2016-87 del 2 de febrero de 2016, art. 2.

51 Zittoun, R., "La sédation continue jusqu'à la mort. Une voie française pour les soins de fin de vie?", La Presse Médicale, vol. 45, n. ${ }^{\circ}$, part 1, 1. ${ }^{\circ}$ de julio, 2016, 670-675.

52 Parlamento Francés, Ley 2016-87 del 2 de febrero de 2016, art. 3. 
El médico tratante debe informar a sus pacientes sobre la posibilidad y las condiciones de redactar directivas anticipadas ${ }^{53}$.

La facultad de designación de un representante está regulada en el artículo 9, el cual señala que esta persona puede acompañar al paciente en el proceso de hospitalización y entrevistas para determinar la historia de valores ${ }^{54}$.

Finalmente, el artículo 10, por influencia directa del caso Lambert ${ }^{55}$, señala:

Cuando una persona se encuentra en la fase avanzada o terminal de un afecto grave e incurable, cualquiera que sea la causa, y no puede expresar su voluntad, el médico tiene la obligación de preguntar sobre la expresión de la voluntad manifestada por el paciente. En ausencia de las directivas anticipadas se debe recopilar el testimonio de la persona de confianza o, en su defecto, cualquier otro testimonio de la familia o parientes.

En Francia, la convocatoria a los estados generales de la bioética durante el año 2018 y el informe del Consejo de Estado respecto de la revisión de las leyes bioéticas demuestran la preocupación por el alto número de muertes de ciudadanos franceses en instituciones de cuidados geriátricos, lo que puede indicar un abandono y rechazo a la vejez ${ }^{56}$. Existe la necesidad de implementar un sistema de acompañamiento más eficaz y de establecer una relación deliberativa entre médico y paciente que permita generar la confianza y así incrementar el uso de directivas anticipadas, las cuales corresponden apenas a un $11 \%$ de los adultos mayores de cincuenta años en ese país ${ }^{57}$.

En Alemania, la ley reformatoria del BGB, del 1. ${ }^{\circ}$ de septiembre de 2009 , fortaleció la regulación de la voluntad anticipada. Para emitir una voluntad anticipada se requiere tener dieciocho años, ser capaz y emitir expresamente el consentimiento o rechazo de un tratamiento referido a un momento futuro en el que no se pueda hacer dicha manifestación de voluntad. Los efectos de las voluntades se producen desde el momento en que la persona carece de capacidad, y las mismas pueden ser revocadas en cualquier momento.

La concentración en el documento ha provocado en Alemania que el uso de las voluntades anticipadas aún sea infrecuente, por lo que los esfuerzos más recientes

53 Parlamento Francés, Ley 2016-87 del 2 de febrero de 2016, art. 8.

54 Parlamento Francés, Ley 2016-87 del 2 de febrero de 2016, art. 9.

55 GARCíA, A., "Apuntes sobre la Ley francesa de 2 de febrero de 2016 por la que se crean nuevos derechos de los enfermos y de las personas al final de su vida y la Sentencia del Tribunal Europeo de Derechos Humanos de 5 de junio de 2015, recaída en el caso Lambert y otros contra Francia", Derecho y Salud, vol. 26, n. ${ }^{\circ}$ extra 1, 2016, 496-501.

56 CONSEIL D’ÉTAT, “Révision de la Loi de Bioéthique: quelles options pour demain?”, [en línea], 2018, 113, disponible en: https://www.conseil-etat.fr/ressources/etudes-publications/rapports-etudes/ etudes/revision-de-la-loi-de-bioethique-quelles-options-pour-demain [consultado el 28 de junio de 2018].

57 Conseil D’ÉtAT, “Révision de la Loi de Bioéthique: quelles options pour demain?”, cit., 117-119. 
se han concentrado en adoptar un sistema de comunicación con el paciente y en la capacitación a los profesionales médicos y de enfermería para informar a los mismos la posibilidad de elaborar dicho documento ${ }^{58}$. La legislación alemana prevé la libre revocabilidad y modificación de las voluntades y prescinde de la formalidad para su suscripción; además, desde el año 2009, la obligatoriedad para el médico es indiscutible; por lo que esta aparente simplificación en la legislación ha resultado más efectiva, especialmente entre las personas mayores ${ }^{59}$.

España introdujo la regulación vigente sobre directivas anticipadas en la Ley 41/2002, de 14 de noviembre, básica reguladora de la autonomía del paciente y de derechos y obligaciones en materia de información y documentación clínica.

El artículo 11 de la citada ley regula las instrucciones previas como el documento por el cual "una persona mayor de edad ${ }^{60}$, capaz y libre, manifiesta anticipadamente su voluntad, con objeto de que ésta se cumpla cuando no sea capaz de expresarla personalmente, sobre los cuidados y el tratamiento de su salud; o, una vez llegado el fallecimiento, sobre el destino de su cuerpo o de los órganos del mismo". El otorgante del documento puede designar un representante para que, llegado el caso, sirva como interlocutor suyo con el médico o el equipo sanitario para procurar el cumplimiento de las instrucciones previas ${ }^{61}$.

En España las instrucciones previas deben constar siempre por escrito y registrarse en la historia clínica. El límite del contenido está dado por el ordenamiento jurídico y la lex artis. El documento puede ser revocado en cualquier momento, aunque no se prevé renovación periódica, lo cual ha sido criticado por la doctrina ${ }^{62}$.

Las instrucciones previas cumplirían los requisitos de un negocio jurídico unilateral celebrado entre el paciente y el médico que además tiene algunos efectos inter vivos, a saber, la mayoría de los relacionados con la autonomía de la voluntad y el poder de representación; y otros post mortem, como los referidos a la donación de órganos.

58 Lupatatzis, B. y Krones, T., "Die Patientenverfügung 'Plus', das Konzept des Advance Care Planning (ACP)", Praxis, vol. 106, n. ${ }^{\circ} 25,2017,1369-1375$.

59 WIESING, U. et al., "A new law on advance directives in Germany", Journal of Medical Ethics, vol. $36, n .^{\circ} 12,2010,79-83$.

60 El artículo 9.3 literal c) de la Ley 41/2002 regula el consentimiento por representación en el caso de niñas, niños y adolescentes y señala que, "cuando éste no tenga la capacidad intelectual y emocional de comprender el alcance de la intervención, el consentimiento debe darlo un representante, previo escuchar al menor". El numeral 4 del artículo 9, por su parte, señala que no se requiere consentimiento por representación en adolescentes que hayan cumplido los dieciséis años y en menores emancipados.

La legislación señalaría en esta norma una presunción de mayoría de edad con el cumplimiento de los dieciséis años; sin perjuicio de que un niño, niña o adolescente no emancipado o menor de dieciséis años pueda ejercer su derecho a la información sanitaria si goza de capacidad intelectual y emocional para su comprensión, lo que será evaluado en el caso concreto con el soporte de los informes periciales o pruebas correspondientes.

61 Jefatura de Estado, Ley 41/2002, 14 de noviembre de 2000.

62 Galán, C., Responsabilidad médica, 2. a ed., Navarra, Thomson Civitas, 2007, 446. 
La amplitud de la redacción de la ley respecto al contenido permitiría a la persona incluir en las instrucciones previas los deseos de rechazar un tratamiento, autorizarlo, solicitar acompañamiento de familiares, designar representante que tome las decisiones por ella, decidir sobre la donación de sus órganos, solicitar cuidados paliativos.

El desarrollo de la legislación en las comunidades autónomas ha sido bastante prolijo $^{63}$, siendo inclusive anterior a la Ley Básica ${ }^{64}$; de la misma manera, ha demostrado algunos avances interesantes respecto a esta, como por ejemplo, la posibilidad de que los menores emancipados puedan suscribir instrucciones previas ${ }^{65}$ o el establecimiento de registros propios de las mismas.

La ley italiana es la de más reciente promulgación entre los países europeos; sin embargo, ya se había acumulado previamente jurisprudencia importante, como la del caso emblemático de Eluana Englaro ${ }^{66}$.

El 31 de enero de 2018 entró en vigor en Italia la Ley 219/2017 que regula el consentimiento informado y las disposiciones anticipadas de tratamiento, primer marco normativo de este país en la materia.

El artículo 4 de la ley en mención permite a "cualquier persona mayor de edad capaz suscribir directivas anticipadas respecto a la autorización o rechazo de tratamientos, pruebas diagnósticas o cualquier tipo de intervención médica". Se regula además el poder de representación a un fiduciario, poder que puede ser revocado en cualquier momento. Hay novedad respecto a la posibilidad de nombrar un administrador de apoyo en caso de falta del fiduciario.

En cuanto a las formalidades, el artículo 4.6 prevé la posibilidad de que la directiva de tratamiento anticipada sea suscrita en escritura pública, ante notario o entregada al registro especial con que cuente la municipalidad. Los documentos descritos pueden ser revocados, modificados o renovados en cualquier momento.

El contenido de las directivas es obligatorio para los profesionales médicos, a menos que se pueda establecer, razonablemente, que las circunstancias en que se

63 Toro, R., "Conocimientos y actitudes de usuarios, médicos y enfermeras sobre las instrucciones previas en el Área Asistencial Este de la Comunidad de Madrid”, cit., 43-54.

64 Ley 21/2000 de Cataluña de 29 de diciembre de 2000 y Ley 3/2001 de Galicia de 28 de mayo de 2001.

65 Ley 5/2003 de Andalucía de 19 de octubre de 2003, art. 4; Ley Foral de Navarra 17/2010 de 8 de noviembre de 2010, art. 54, y Ley 1/2003 de Valencia de 28 de enero de 2003, art. 17.2.

66 El razonamiento del Tribunal Constitucional italiano en este caso señala que el juez puede autorizar la desactivación de la hidratación y alimentación artificial del paciente en estado de inconsciencia cuando el estado vegetativo sea, de acuerdo con un riguroso dictamen clínico, irreversible, y no exista fundamento médico, según los estándares científicos reconocidos a nivel internacional, que permita suponer la mínima posibilidad de alguna recuperación de la conciencia y retorno a la percepción del mundo exterior. Véase, en profundidad, Veronesi, P., "Diritto di vivere, libertà di morire? Il caso di Eluana Englaro", [en línea], en Presno LinERA, M. (coord.), Autonomía personal, cuidados paliativos y derecho a la vida, Oviedo, Universidad de Oviedo, 2011, 129 ss., disponible en: https://www.personaedanno.it/articolo/diritto-di-vivere-liberta-di-morire-il-caso-di-eluanaenglaro-paolo-veronesi [consultado el 4 de marzo de 2020]. 
van a aplicar son distintas a aquellas en que fueron emitidas, o exista una terapia de carácter curativo respecto al diagnóstico ${ }^{67}$.

No existe referencia directa a la situación de los adultos mayores; sin embargo, el enfoque contenido en los artículos 1.10 y 4 otorga una regulación favorable para la atención integral a las personas mayores respecto al final de la vida.

La figura de las directivas anticipadas ha tenido menor regulación en Latinoamérica, en donde ha sido incluida solamente en los ordenamientos jurídicos de cuatro países; de la misma manera, se verifican disímiles estados de desarrollo en estos. Antes de reflexionar sobre el caso chileno, en el siguiente acápite se revisan brevemente los casos de la región.

\section{Los pioneros en Latinoamérica}

La discusión de la materia en Colombia surge a partir de la sentencia de la Corte Constitucional C-239 de 1997 sobre el homicidio por piedad.

La alusión de la Corte a la necesidad de que el legislador regule el derecho a la muerte digna y la forma en que ese consentimiento sea manifestado es acogida en la Ley 1733 de 2014 que regula el servicio de cuidados paliativos y la atención de enfermedades terminales, crónicas, degenerativas e irreversibles. Este cuerpo legal señala en su artículo 5 numeral 4 que "toda persona capaz, sana o en estado de enfermedad, en pleno uso de sus facultades legales y mentales y con total conocimiento de las implicaciones que acarrea el presente derecho podrá' suscribir el documento de voluntad anticipada". No se hace mención directa al grupo de las personas mayores $^{68}$.

La sentencia de la Corte Constitucional T-970 de 2014, expedida con ocasión de una acción de tutela en la que se reclama el derecho a morir dignamente, ordena al Ministerio de Salud emitir una directriz a todos los prestadores del servicio de salud para que conformen un grupo interdisciplinario de expertos para que cumpla varias funciones cuando se esté en presencia de casos en los que se solicite el derecho a morir dignamente ${ }^{69}$.

Como resultado de la disposición contenida en la sentencia, el Ministerio de Salud expidió la Resolución 1216 del 21 de abril de 2015 que regula los comités

67 Parlamento italiano, Ley 219/2018, 31 de enero de 2018, art. 4.

68 En resumen, los requisitos de las voluntades anticipadas en Colombia contenidos en la Ley 1733/2014 son: "1) deben provenir de una persona mayor de 18 años, competente, que puede encontrarse sana o aquejada de una enfermedad terminal o crónica; 2) debe estar precedida de información, la cual incluye los derechos que tienen las personas al final de la vida; 3) su objeto debe ser lícito, incluido el rechazo a tratamientos fútiles y a la donación de órganos, y 4) la exteriorización de la voluntad debe ser expresa y por escrito". Bolívar, P. L. y GómEz, A. I., "Voluntades anticipadas al final de la vida. Una aproximación desde la regulación colombiana y en el derecho comparado", Revista Latinoamericana de Bioética, vol. 16., n. ${ }^{\circ} 30-1,2015,141$.

69 Corte Constitucional de Colombia. Sentencia T-970 de 2014. 
científicos interdisciplinarios para el derecho a morir con dignidad. Respecto a las voluntades anticipadas, el artículo 15 señala que el consentimiento para que se dé trámite a una solicitud de muerte anticipada debe ser expresado de manera libre e informada. El consentimiento puede ser previo a la enfermedad terminal cuando el paciente haya manifestado, antes de la misma, su voluntad en tal sentido.

El camino uruguayo sobre la materia se concreta en la Ley 18.473 publicada el 21 de abril de 2009, cuya entrada en vigor fue postergada hasta su reglamentación contenida en el Decreto 385-2013 del 4 de diciembre de 2013.

En el artículo 1, la ley en cita parte de la capacidad de la persona que emite las voluntades, y considera el aspecto etario y psíquico. Se atiende en primer lugar el derecho a rechazar tratamientos o procedimientos médicos, como parte del consentimiento informado; para posteriormente abarcar el derecho a señalar voluntades anticipadas respecto a tratamientos destinados a la prolongación de la vida en detrimento de su calidad ${ }^{70}$.

La imprecisión respecto a definir la aptitud psíquica ha sido señalada como una deficiencia del legislador que genera ambigüedad ${ }^{71}$; adicionalmente, el factor etario rígido señalado en el reglamento limita la posibilidad de que el derecho pueda ser ejercido por menores adultos, o de que se tomen en cuenta principios como la capacidad progresiva y el interés superior del niño.

El artículo 6 de la ley prevé la posibilidad de designar un representante y el artículo 7 establece una prelación de la representación, en caso de que no se haya designado a nadie: el cónyuge o concubina ${ }^{72}$ tiene prioridad en primer lugar, y en segundo lugar, los familiares de primer grado.

En Argentina, el desarrollo legislativo de las directivas anticipadas sucedió primero en las provincias ${ }^{73}$, antes de llegar a consolidarse la Ley Federal 26.742 del 24 de mayo de 2012, que a su vez modificó la Ley 26.529 del 19 de noviembre de 2009 que regula los derechos de los pacientes en su relación con los profesionales e instituciones de la salud. Finalmente, el Código Civil y Comercial de la Nación promulgado el 8 de octubre de 2014 incorpora las directivas médicas anticipadas ${ }^{74}$.

La modificación que introduce la Ley 26.742 tiene la virtud de especificar lo que el paciente puede autorizar o rechazar en la directiva anticipada, refiriéndose expresamente a procedimientos quirúrgicos, reanimación artificial y retiro de medidas de soporte vital cuando sean desproporcionadas, extraordinarias respecto a la perspectiva de mejora o produzcan sufrimiento desmesurado.

70 Congreso de la República de Uruguay, Ley 18.473, 21 de abril de 2009.

71 Benítez, R., "Las voluntades anticipadas en Uruguay: reflexiones sobre la Ley 18473", Revista IUS, vol. 9 , n. ${ }^{\circ} 36,2015,145-146$.

72 Ibíd., 152.

73 Monteiro, R. y Silva Junior, F., "Directivas anticipadas de voluntad: recorrido histórico en América Latina”, Revista Bioética, vol. 27, n. ${ }^{\circ}$ 1, 2019, 91 .

74 Congreso de la República Federal de Argentina, Código Civil y Comercial de la Nación, 8 de octubre de 2014. 
El artículo 11 de la Ley 26.529 señala que "toda persona capaz y mayor de edad puede emitir directivas anticipadas". El contenido debe versar sobre el consentimiento o rechazo de tratamientos médicos o cuidados paliativos, aunque se deja abierta la posibilidad de plasmar cualquier otra decisión sobre la propia salud ${ }^{75}$.

La norma señalada otorga obligatoriedad a las declaraciones anticipadas respecto del médico tratante y entiende como no escrita cualquier prescripción eutanásica.

México aún no ha ratificado o se ha adherido a la CIDHPM, por lo que la fuente formal respecto a la materia se mantiene en la Ley General de Salud. El artículo 166 bis 4 regula directamente las voluntades anticipadas, definidas como el derecho que tiene:

... toda persona mayor de edad, en pleno uso de sus facultades mentales[,] para, en cualquier momento e independientemente de su estado de salud, expresar su voluntad por escrito ante dos testigos, de recibir o no cualquier tratamiento, en caso de que llegase a padecer una enfermedad y estar en situación terminal y no le sea posible manifestar dicha voluntad. El documento en el que se haga constar la voluntad es revocable en cualquier momento.

Finalmente, en México, la primera ley en regular las voluntades anticipadas, a nivel estatal, fue la del Distrito Federal, publicada el 7 de enero de 2008 y reformada en el año 2012. Esta ley señala el derecho de toda persona a ser sometida o no a medios, tratamientos o procedimientos médicos que pretendan prolongar su vida cuando se encuentre en etapa terminal y, por razones médicas, sea imposible mantenerla de manera natural, protegiendo en todo momento la dignidad de la persona ${ }^{76}$.

Finalizada esta breve revisión sobre la regulación de las directivas anticipadas en la región, a continuación se reseñan los principales hitos en el caso chileno, por cuanto no existe normativa expresa que se haya adoptado, aunque sí proyectos en proceso y rezagos de un intento fallido en la actual Ley 20.584 que rige los derechos y deberes de las personas en relación con acciones vinculadas a su atención en salud.

\section{Chile y sus oportunidades}

Según los datos extraídos del Censo 2017, Chile tiene una población de personas mayores que equivale al $11 \%$ del total. Este porcentaje corresponde al doble de lo que representaba la población de personas mayores en 1992, habiéndose pasado en quince años de 877.046 a 2.003.256. Estos datos no solo demuestran una tendencia sostenida de envejecimiento de la sociedad chilena, sino además una etapa avanzada

75 Congreso de la República Federal de Argentina, Ley 26.529 del 19 de noviembre de 2009.

76 Asamblea Legislativa del Distrito Federal, Ley de Voluntades Anticipadas para el Distrito Federal del 7 de enero de 2008, art. 1 . 
de la transición demográfica ${ }^{77}$. Adicionalmente Chile sería una de las primeras sociedades envejecidas de Latinoamérica ${ }^{78}$.

Por otro lado, el maltrato a las personas mayores ha sido identificado como estructural, es decir, producido dentro de la sociedad; y, multidimensional, por lo que puede manifestarse en el ámbito social, económico, cultural o político ${ }^{79}$.

Respecto a la salud, las personas mayores son vulnerables, por cuanto el requerimiento de atención es más frecuente y sufren en mayor medida de enfermedades complejas, incurables e irreversibles.

Solo en materia de cuidado, el remplazo de la familia por la internación en los establecimientos de larga estadía para adultos mayores hace patente la necesidad de que los profesionales de la salud que laboran en estas instituciones adquieran experticia en habilidades comunicativas, conocimiento sobre los derechos de la persona mayor al final de la vida e inclusión de la familia en la toma de decisiones. Lo señalado los convierte en actores importantes en la política pública ${ }^{80}$.

La Ley 20.584 de $2012^{[81]}$, que regula los derechos y deberes que tienen las personas respecto a las acciones vinculadas a su atención en salud, establece una normativa amplia respecto al deber de informar del médico ${ }^{82}$, el acceso a la información por parte del paciente ${ }^{83}$ y el consentimiento informado ${ }^{84}$.

El artículo 16 de dicha ley señala que "la persona que fuere informada de que su estado de salud es terminal tiene derecho a otorgar o denegar su voluntad para someterse a cualquier tratamiento que tenga como efecto prolongar artificialmente su vida, sin perjuicio de mantener las medidas de soporte ordinario. En ningún caso, el rechazo de tratamiento podrá implicar como objetivo la aceleración artificial del proceso de muerte".

La normativa señalada, a pesar de no regular expresamente la posibilidad del paciente de suscribir directivas anticipadas, no impide que se lo haga, como si fuese una extensión del derecho al consentimiento informado.

77 Instituto Nacional de Estadísticas, Síntesis de Resultados Censo 2017, cit.

78 Huenchuan, S., Envejecimiento, personas mayores y Agenda 2030 para el Desarrollo Sostenible: perspectiva regional y de derechos humanos, cit., 34-36.

79 Riveros, C. et al., "El maltrato estructural a persona mayores en Chile y la necesidad de formular un índice multidimensional”, Revista Universum, vol. 32, n. ${ }^{\circ} 2,2017,166$.

80 Lathrop, F., "Protección jurídica de los adultos mayores en Chile", cit., 92-96.

81 Congreso de la República de Chile, Ley 20.584 de 24 de abril de 2012, art. 16.

82 Los artículos 8, 9, 10 y 11 de la Ley 20.584 de 24 de abril de 2012, de Chile regulan los puntos sobre los que el médico debe compartir información suficiente, veraz, oportuna y comprensible respecto a atenciones de salud, condiciones previsionales, derechos y obligaciones del paciente. Se regulan además las obligaciones de identificación del personal médico, información sobre el diagnóstico, pronóstico, alternativas de tratamiento, riesgos y consecuencias de las intervenciones médicas. Se prevé además la obligación del médico de proporcionar informes y garantizar el acceso a la historia clínica y demás información relevante.

83 Regulado en los artículos 12 y 13 de la Ley 20.584, 24 de abril de 2012, de Chile.

84 El artículo 14 contiene el derecho del paciente de rechazar un tratamiento. 
El título del numeral 2 del párrafo 7 de la Ley 20.584 hace alusión al término "voluntad manifestada previamente", sin que el articulado trate la figura. Esto se explica porque el proyecto de ley regulaba el testamento vital ${ }^{85}$.

La lamentable decisión de eliminar esta regulación en el proceso de tramitación de la ley no solo ha dejado como huella la mencionada incongruencia respecto al título del numeral, sino también la dificultad de ejercer el derecho del paciente a emitir voluntades anticipadas ${ }^{86}$.

El desarrollo doctrinario en Chile ha sido abordado desde el análisis del derecho al consentimiento informado ${ }^{87}$.

El consentimiento informado, como manifestación de la autodeterminación del paciente sería una figura novedosa para el ordenamiento jurídico chileno que trajo la Ley $20.584^{[88]}$, ante una práctica médica y jurisprudencial apegada a la conservación de la vida, incluso frente a los deseos expresos señalados por el paciente ${ }^{89}$.

Las limitaciones al consentimiento informado establecidas en la ley generan una regulación tímida hacia la autonomía del paciente. Hubiera sido deseable que

85 El artículo 18 del proyecto de ley original permite indagar sobre la estructura que se proponía para la institución; en primer lugar, esta versaba sobre la voluntad respecto a la práctica de un procedimiento o tratamiento médico, la cual debía concederse ante ministro de fe, y sus efectos solo llegaban a producirse si la persona que la había emitido se encontraba en estado terminal, era incapaz de manifestar su voluntad y su representante no podía hacerlo por ella.

Se prevé en el artículo 18 del proyecto que el contenido de las voluntades anticipadas pueda versar sobre la decisión respecto a donación de órganos, la designación de un representante para la toma de decisiones y la confidencialidad de datos de la ficha clínica. Se señala expresamente que la limitación del contenido de las voluntades anticipadas es el ordenamiento jurídico y el arte médico, emulando al texto de la Ley Básica española; y que, en caso de dudas, se deberá remitir al comité de ética de la institución para su evaluación. Finalmente, se prevé la posibilidad de revocación en cualquier momento, y de la obligatoriedad de su constancia por escrito.

Véase Presidente de la República de Chile, Mensaje 223-354 del 26 de julio de 2006, [en línea], disponible en: http://www.senado.cl/appsenado/templates/tramitacion/index.php?boletin_ini=439811 [consultado el $1 .^{\circ}$ de agosto de 2019].

86 Centro de Estudios y Asistencia Legislativa, Estudio sobre brechas legales en Chile para la implementación de la Convención Interamericana sobre la Protección de los Derechos Humanos de las Personas Mayores, [en línea], Valparaíso, Pontificia Universidad Católica de Valparaíso, 2017, 98, disponible en: http://www.senama.gob.cl/storage/docs/ESTUDIO_SOBRE_BRECHAS_LEGALES_PARA_ CHILE\%2C_IMPLEMENTACION_CIPDHPM.pdf [consultado el 01 de agosto de 2019].

87 Riveros, C. y Barcia, R., "Obligación ética y jurídica de prevenir y reducir al máximo la aparición de nuevas discapacidades en las personas mayores en Chile”, Acta Bioethica, vol. 21, n. ${ }^{\circ} 2,2015$, 207-215.

88 Antes de la Ley 20.584 el deber de información del médico en la relación clínica estaba regulado, para el sector público, en el artículo 34 del Reglamento Orgánico de los Servicios de Salud contenido en el Decreto n. 140 del Ministerio de Salud del 21 de abril de 2005; en el Decreto Supremo n. 570 del Ministerio de Salud, que recoge el Reglamento para la Internación de Personas con Enfermedades Mentales, y en el Código de Ética del Colegio Médico vigente desde el año 2004. La normativa señalada regula el consentimiento informado, su contenido y el derecho del paciente a rechazar un procedimiento diagnóstico o terapéutico, por sí mismo o a través de su representante; sin embargo, no existe mención a la figura de las voluntades anticipadas.

89 ZúÑIGA, A, "La nueva ley de derechos del paciente: del modelo de la beneficencia al modelo de autonomía”, Revista Médica de Chile, vol. 141, 2013, 123. 
el legislador previera con mayor amplitud las distintas circunstancias que pueden producirse alrededor de la interrupción de un tratamiento en curso, especialmente para personas que ya no pueden manifestar su voluntad ${ }^{90}$.

La obligación de recurrir al comité de ética institucional de manera obligatoria y la posibilidad de solicitar el alta voluntaria o ejercicio del derecho a la objeción de conciencia del médico son situaciones que podrían persuadir al paciente de desistir de cualquier decisión que implique negación a un tratamiento ${ }^{91}$. Adicionalmente, la salud pública, el riesgo vital y la imposibilidad de obtener la voluntad del paciente o de un representante limitan el ejercicio del consentimiento al rechazo de tratamientos $^{92}$.

Ante estas dificultades, al paciente le quedaría la opción del acceso a los cuidados paliativos hasta que se produzca la muerte de manera natural; sin embargo, esto conllevaría una doble vulneración: por un lado, a su autodeterminación, al no materializar sus deseos y preferencias; por el otro, a su dignidad, al obligarlo a esperar la hora de su muerte natural, muchas veces cuando los cuidados paliativos son ineficaces ante el dolor.

Respecto a las voluntades anticipadas, su posibilidad hipotética de contenido se reduciría aún más, puesto que la Ley 20.584, como se ha señalado, contiene imprecisiones respecto al ejercicio del derecho a rechazar un tratamiento que alargue la vida de manera artificial.

Un proyecto de ley entregado por el Presidente de la República a la Cámara de Diputados, mediante Mensaje 015-367 del 27 de marzo de 2019, tiene como fin regular el acceso a cuidados paliativos; sin embargo, en el numeral 4 del artículo 3 tan solo se enuncian las voluntades anticipadas, sin proceder a regularlas. Por lo tanto, esta mención reitera el error sobre su aparición sin contenido en la Ley 20.584, provocado por la eliminación de la figura en su proceso de tramitación ${ }^{93}$.

Ante el reto de regular específicamente las directivas anticipadas en Chile, tanto por el progresivo envejecimiento poblacional como por las obligaciones contraídas con la ratificación de la CIDHPM, en el siguiente apartado se profundiza en el modelo de planificación anticipada del cuidado en salud, como una posibilidad de tratamiento integral de la laguna legislativa.

90 Riveros, C. y BARCIA, R., “Obligación ética y jurídica de prevenir y reducir al máximo la aparición de nuevas discapacidades en las personas mayores en Chile", cit., 208.

91 Figueroa, R., "Consentimiento informado en la nueva Ley de Derechos de los Pacientes", Revista Médica de Chile, vol. 140, 2012, 1349.

92 Ossandón, M., "El derecho a rechazar tratamientos médicos ¿un reconocimiento del derecho a disponer de la propia vida?", Revista Derecho Público Iberoamericano, n. ${ }^{\circ} 2,2013,155$.

93 Véase Presidencia de la República de Chile, Mensaje 015-367 del 27 de marzo de 2019, artículo tercero, numeral cuatro. 


\section{La planificación anticipada del cuidado en salud en las personas adultas mayores}

Las constataciones del informe S.U.P.P.O.R.T. respecto a la necesidad de una mayor eficacia de las directivas anticipadas permitieron concluir que debe existir formación del profesional médico respecto a sus deberes de información con el paciente, así como sobre sus derechos, y llevarse a cabo una comunicación constante que genere confianza.

La familia y el entorno del paciente deben involucrarse, y el representante para la toma de decisiones necesita conocer a fondo la voluntad de este para hacerla prevalecer en el caso que corresponda; y la historia de valores del paciente debía ser clara y quedar registrada como base de la interpretación de las directivas anticipadas ${ }^{94}$.

Ezekiel y Linda Emanuel describieron cuatro modelos de relaciones entre médico y paciente ${ }^{95}$. Se concuerda con los autores en que el modelo deliberativo es el más adecuado respecto de los derechos al final de la vida y de la planificación anticipada del cuidado en salud, por cuanto se requiere que el papel del médico refleje una toma de posición sobre recomendaciones expresas con base en su experiencia.

Partiendo del enfoque deliberativo, se puede definir el modelo de planificación anticipada del cuidado en salud como el proceso por el cual se garantiza que el consentimiento del paciente se cumpla, estableciendo las principales decisiones sobre su cuidado de salud en constante comunicación con el equipo médico tratante, su familia y otras personas relevantes de su entorno, en un contexto interdisciplinario ${ }^{96}$. Las directivas anticipadas forman parte de la planificación y su función es registrar y proporcionar obligatoriedad jurídica a la voluntad del paciente ${ }^{97}$.

La planificación anticipada del cuidado en salud ha resultado exitosa, tanto en la percepción del paciente respecto a la calidad de la atención como en lo que hace al bienestar de la familia y el representante tomador de decisiones. Se ha comprobado reducción del estrés y mayor resiliencia en las familias que han sido parte de este

94 JimenEZ, G. et al., "Overview of systematic reviews of advance care planning: summary of evidence and global lessons", Journal of Pain and Symptom Management, vol. 56, n. ${ }^{\circ}$, 2018, 454-456.

95 Emanuel, E. y Emanuel, L., "Four models of the physician-patient relationship", JAMA, vol. 267, n. ${ }^{\circ} 16,1992,2221-2223$. El primer modelo es el paternalista, en el que prima el criterio del médico sobre el mejor interés del paciente sin participación de este; el segundo el informativo, en el que el médico tiene el deber de proporcionar al paciente toda la información de la manera más certera posible, y este último deberá elegir entre las opciones de tratamiento informadas. Los dos modelos señalados serían los extremos de paternalismo y autonomía; a partir de ellos surgen otros eclécticos, como el interpretativo, en el cual el profesional médico tiene como objetivo interpretar los valores y preferencias del paciente para actuar en función de ellos; así como las intervenciones que el paciente autorice. Finalmente, el modelo deliberativo se desarrolla a través de consensos, donde el médico puede persuadir al paciente sobre determinadas opciones, pero en el caso de ser rechazadas, actuará según los deseos del paciente.

96 Singer, P. et al., "Bioethics for clinicians: 6. Advance care planning", cmaJ: Canadian Medical Association Journal, vol. 155, n. ํ 12, 1996, 1689-1690.

97 Ibíd., 1691-1692. 
proceso, una vez que el paciente ha fallecido ${ }^{98}$. Si bien la autodeterminación del paciente es un derecho fundamental y objetivo primordial de la primera etapa de desarrollo de las directivas anticipadas, ahora son la calidad del cuidado y el bienestar los elementos que adquieren mayor relevancia ${ }^{99}$.

Cambiar el foco de atención desde el documento hacia el proceso de atención integral con garantía de los derechos de la persona al final de la vida es fundamental para la eficacia de la planificación anticipada del cuidado en salud.

Respecto a la población mayor es clave insertar la planificación en el momento en que la persona aún se encuentra en plena capacidad de tomar decisiones, por lo que el trabajo debe iniciar cuando la persona es plenamente capaz de decidir ${ }^{100}$; en segundo lugar, la atención primaria y los establecimientos de larga estadía deben incorporar un equipo interdisciplinario que se encargue de elaborar la planificación con la inclusión de los familiares y el entorno cercano a la persona mayor.

El trabajo ideal consiste en alcanzar la normalización de la conversación sobre el cuidado al final de la muerte entre médico, familia y paciente, así como en la consolidación de este debate como parte de la cultura a través de la difusión de la información por medios tecnológicos y con equipos interdisciplinarios ${ }^{101}$.

El sistema requiere tiempo para ser conocido por parte de los pacientes, razón por la cual en Estados Unidos ha tomado casi tres décadas (desde la promulgación de la Patient Self Determination Act) para que entre el $47 \%$ y el $72 \%$ de la población de personas mayores llegara a hacer uso de la institución de la planificación anticipada en materia de salud, en contraste con países latinoamericanos o España donde es prácticamente desconocida o presenta un porcentaje de uso mínimo ${ }^{102}$.

La implementación de la planificación entre las personas mayores requiere la comprensión previa del enfoque deliberativo de la relación entre médico y paciente ${ }^{103}$, así como la implementación de una política pública amplia que la introduzca como un valor cultural y normalice la comunicación al respecto. En la atención

98 Detering, K. et al., "The impact of advance care planning on end of life care in elderly patients: randomised controlled trial", $B M J$, vol. 340, 2010, 1-9.

99 Johnson, S. et al., "Advance care planning: is quality end of life care really that simple?", Internal Medicine Journal, vol. 47, n. ${ }^{\circ}$ 4, 2016, 391.

100 Piers, R. et al., "Advance care planning in dementia: recommendations for healthcare professionals", вмC Palliative Care, vol. 17, n. ${ }^{\circ}$ 1, 2018, 14-15.

101 Canadian Hospice Palliative Care Association, Advance Care Planning in Canada, cit., 23-24

102 Kernell-Schiffman, I. y Werner, P., "Knowledge regarding advance care planning: A systematic review", Archives of Gerontology and Geriatrics, vol. 73, 2017, 140.

103 Cabe aclarar que el enfoque deliberativo es esencial para un proceso comunicativo y a largo plazo como la planificación anticipada del cuidado en salud, pero no significa que se deban descartar las otras perspectivas; es así que un enfoque paternalista será esencial en un momento de urgencia médica con peligro de vida, o un enfoque informativo podrá ser aplicado en medicina voluntaria. Emanuel, E. y Emanuel, L., "Four models of the physician-patient relationship", cit., 2225-2226. 
de personas mayores el valor de la conversación y el trabajo a largo plazo son esenciales $^{104}$.

Una propuesta de regulación puede elaborarse a partir de las recomendaciones sobre planificación anticipada del cuidado en salud dispuesta como un proceso de ocho fases propuesto por Ruth Piers ${ }^{105}$.

El proceso de planificación inicia cuando el paciente ha comprendido las implicaciones de un diagnóstico irreversible y la importancia de planificar, existiendo la posibilidad de cubrir el proceso de manera integral respecto a los intereses del paciente al final de la vida, conjuntamente con la familia y cuidadores informales ${ }^{106}$.

Se debe iniciar la planificación lo más pronto posible en el cuidado de salud diario. Dos momentos son centrales para iniciar: el primero, cerca del diagnóstico de disminución o ausencia de la capacidad; el segundo, cuando el paciente esté convencido de la importancia de la planificación.

Para la evaluación de la capacidad se recomienda determinar, en primer término, los deseos y preferencias, estableciendo de la manera más clara posible los valores y creencias espirituales. La capacidad debe ser evaluada constantemente por un equipo interdisciplinario.

La siguiente fase es el inicio del proceso comunicativo mismo, que debe ser adaptado al paciente e involucrar a las personas que este considera importantes ${ }^{107}$. Se deben perseguir las metas de la planificación en el respeto de los valores, normas y creencias.

La familia y el entorno más cercano requieren de apoyo del equipo para actualizar la historia de valores. Se debe procurar que el representante elegido para la toma de decisiones esté presente en todo el proceso ${ }^{108}$.

La fase final debe concentrarse en el registro de información, decisiones sobre el final de la vida y formación del equipo interventor.

Como se puede observar, el proceso reseñado implica tiempo, dedicación, preparación y recursos; sin embargo, tiene sin duda efectos importantes en la garantía de la dignidad del paciente. El sostenimiento de la planificación debe contar con un marco jurídico amplio en lo referente a la consignación legal de las recomendaciones.

104 Lum, H. et al., "A group visit initiative improves advance care planning documentation among older adults in primary care", JABFM. Journal of the American Board of Family Medicine:, vol. 30, n. ${ }^{\circ} 4$, 480-481.

105 Piers, R. et al., “Advance care planning in dementia”, cit., 8-17.

106 Piers, R. et al., "Advance care planning in terminally ill and frail older persons", Patient Education and Counseling. Quality of Communication from the Patient Perspective, vol. 90, n. ${ }^{\circ}$, 2013, 327328.

107 Piers, R. et al., “Advance care planning in dementia”, cit., 11-12.

108 Abu Al Hamayel, N. et al., "Preparing older patients with serious illness for advance care planning discussions in primary care", Journal of Pain and Symptom Management, 7 de mayo, 2019, 3-7. 


\section{Conclusiones}

En el ámbito de salud, la CIDHPM fortalece la autonomía de la persona mayor, a través de la regulación de los requisitos y el contenido del consentimiento informado respecto a tratamientos y procedimientos médicos. Es el primer instrumento internacional multilateral de derechos humanos en la región que regula las voluntades anticipadas como derecho de las personas mayores, por lo que, para aquellos países que lo han ratificado, existe la obligación de adecuar su ordenamiento jurídico al mismo.

La autodeterminación del paciente fue el fundamento de la aparición de la institución del testamento vital y de su evolución progresiva en la legislación y la jurisprudencia en Estados Unidos, especialmente en lo relativo al derecho al rechazo de tratamientos que puedan identificarse con el encarnizamiento terapéutico y el soporte de la vida no curativo.

La primera etapa del desarrollo normativo sobre directivas anticipadas tiene una acentuada concentración en el instrumento legalmente vinculante y el perfeccionamiento del poder de representación para la toma de decisiones por el paciente.

El modelo de planificación anticipada del cuidado en salud ha sido el principal objeto de estudio de los últimos veinte años en el ámbito anglosajón y en Europa occidental, desarrollando principios para una atención médica centrada en el paciente, su historia de valores, la integración de la familia, los cuidados paliativos y la planificación de las decisiones sobre el final de la vida en los momentos en que aún existe plena capacidad.

En Latinoamérica, Colombia, Uruguay, Argentina y México han legislado sobre voluntades anticipadas. Estos cuatro países han enfocado su legislación en el documento que contiene las directivas y, en menor grado, en el proceso.

En Argentina, la legislación aclara expresamente que la alimentación y la hidratación artificial constituyen un procedimiento que, sin tener un fin curativo, está destinado únicamente a alargar la vida y, por ende, puede rechazarse. Todas las legislaciones prevén que las directivas anticipadas puedan revocarse en cualquier momento, aunque no prevén renovaciones periódicas. Ninguna legislación abarca de manera especializada a las personas mayores, por lo que la CIDHPM incorporaría una preocupación adicional que debe ser abarcada por la legislación o la política pública.

La ratificación de la CIDHPM por parte de Chile incorpora en el ordenamiento jurídico chileno el derecho de las personas mayores a emitir directivas anticipadas, esto en razón de la disposición contenida en el artículo 11 último inciso de la Convención y de la prescripción contenida en el artículo 5 inciso segundo de la Constitución de la República ${ }^{109}$.

A pesar de lo señalado, la ausencia de legislación específica genera interrogantes sobre qué formalidades son necesarias para su validez, contenido, nombramiento de

109 Riveros, C., "El maltrato estructural a persona mayores en Chile y la necesidad de formular un índice multidimensional", cit., 4. 
representante, renovación y revocatoria; pero, la mayor dificultad se produce respecto a la contraposición entre la voluntad de interrupción de un tratamiento destinado a prolongar la vida sin fin terapéutico y la prohibición expresa de prácticas eutanásicas o que aceleren la muerte contenida en la Ley 20.584. El caso del paciente en estado vegetativo permanente sobreviniente tampoco tendría respuesta.

Se considera sin embargo que la hipótesis acá planteada se corrobora, por cuanto, hasta la regulación específica de la institución en la ley, permanece la norma de la Convención como compromiso cuyo incumplimiento acarrearía responsabilidad internacional. Desde este punto de vista, la Convención es un mínimo de regulación, por lo que constituye un punto de partida en el desarrollo en Chile de la institución en estudio. Por lo pronto, como se puede observar, a la fecha del presente trabajo no existe ley ni proyecto de ley que aborde las voluntades anticipadas de manera expresa e integral en Chile. Y tampoco se cuenta con una ley específica que regule los derechos de las personas mayores ${ }^{110}$. A la luz de lo anterior, es de señalar que la oportunidad de regular la institución en el proyecto de ley que está en trámite es enorme si existe la voluntad de consolidar la figura y proteger la autonomía del paciente persona mayor, alineándose con los compromisos adquiridos con la ratificación de la Convención.

\section{Referencias}

Abu Al Hamayel, N. et al. " Preparing older patients with serious illness for advance care planning discussions in primary care", Journal of Pain and Symptom Management, 7 de mayo, 2019, 1-9.

AdiB, P., "Comentarios a la Ley de Voluntad Anticipada para el Distrito Federal", Boletín Mexicano de Derecho Comparado, vol. 41, n. ${ }^{\circ}$ 123, 2008, 1533-1556.

Altisent, R. y JúDEZ, J., "El reto de la planificación anticipada de la atención al final de la vida en España", Medicina Paliativa, vol. 23, n. ${ }^{\circ}$ 4, 2016.

Arbe, M. M., "Estudio de las instrucciones previas en el ámbito sanitario a través del ordenamiento jurídico español”, tesis de doctorado, Salamanca, Universidad de Salamanca, 2013.

110 El Servicio Nacional del Adulto Mayor, creado por la Ley 19.828 del 17 de septiembre de 2002, puede promover un programa de planificación anticipada del cuidado en salud en la atención primaria y en los Establecimientos de Larga Estadía para los Adultos Mayores. Dicho programa puede ser implementado por este organismo dentro de sus facultades. 
Arimany-Manso, J., "El testamento vital o documento de voluntades anticipadas. Consideraciones médico-legales y análisis de la situación de implantación en España”, Revista Española de Medicina Legal, vol. 43, n. 1, 2017, 35-40.

Benítez, R., "Las voluntades anticipadas en Uruguay: reflexiones sobre la Ley 18473", Revista IUS, vol. 9, n. ${ }^{\circ} 36,2015,135-154$.

Bolívar, P. L. y Gómez, A. I., "Voluntades anticipadas al final de la vida. Una aproximación desde la regulación colombiana y en el derecho comparado", Revista Latinoamericana de Bioética, vol. 16, n. ${ }^{\circ}$ 30-1, 2015, 128-153.

Boulanger, A., "Les directives anticipées et le désir de maîtrise de sa fin de vie", Médicine \& Droit, 2017, 136-140.

Canadian Hospice Palliative Care Association, Advance Care Planning in Canada. National Framework, [en línea], 2012, 1-29, disponible en: http://www.advancecareplanning.ca/wp-content/uploads/2016/08/ACP-Framework-2012-ENG.pdf [consultado el 18 de julio de 2019].

Carrasco, V. H. y CrisPi, F., "Eutanasia en Chile: una discusión pendiente”, Revista Médica de Chile, vol. 144, n. ${ }^{\circ} 12,2016,1598-1604$.

Centro de Estudios y Asistencia Legislativa, Estudio sobre brechas legales en Chile para la implementación de la Convención Interamericana sobre la Protección de los Derechos Humanos de las Personas Mayores, [en línea], Valparaíso, Pontificia Universidad Católica de Valparaíso, 2017, disponible en: http://www. senama.gob.cl/storage/docs/ESTUDIO_SOBRE_BRECHAS_LEGALES_PARA_CHILE\%2C_ IMPLEMENTACION_CIPDHPM.pdf [consultado el 1 de agosto de 2019].

Connors, A. et al., "A controlled trial to improve care for seriously III hospitalized patients: the Study to Understand Prognoses and Preferences for Outcomes and Risks of Treatments (SUPPORT)", JAMA, vol. 274, n. ${ }^{\circ} 20,1997,1591-1598$.

Dabove, M., “Autonomía y vulnerabilidad en la vejez: respuestas judiciales emblemáticas", Revista de Derecho Privado, Universidad Externado de Colombia, vol. 34, marzo, 2018, 53-85.

Dabove, M., "Derechos humanos de las personas mayores en la nueva Convención Americana y sus implicancias bioéticas", Revista Latinoamericana de Bioética, 16, n. $^{\circ} 1,2016,38-59$. 
Emanuel, E. y Emanuel, L., "Four models of the physician-patient relationship", JAMA, vol. 267, n. ${ }^{\circ} 16,1992,2221-2226$.

FigueroA, R., "Consentimiento informado en la nueva Ley de Derechos de los Pacientes", Revista Médica de Chile, vol. 140, 2012, 1347-1351.

FLores, L., "Autonomía y manifestación de la voluntad en el testamento vital y documento de voluntad anticipada en México", Revista IUS, vol. 9, n. ${ }^{\circ} 36,2015$, 155-178.

Galán, C., Responsabilidad Médica, 2. a ed., Navarra, Thomson Civitas, 2007.

GARcía, A., "Apuntes sobre la Ley francesa de 2 de febrero de 2016 por la que se crean nuevos derechos de los enfermos y de las personas al final de su vida y la Sentencia del Tribunal Europeo de Derechos Humanos de 5 de junio de 2015, recaída en el caso Lambert y otros contra Francia”, Derecho y Salud, vol. 26, n. ${ }^{\circ}$ extra 1, 2016, 496-501.

Heywood, R., "Revisiting advance decision making under the Mental Capacity Act 2005: a tale of mixed messages", Medical Law Review, vol. 23, n. 1, 2015, 81-102.

Huenchuan, S. (ed.), Envejecimiento, personas mayores y Agenda 2030 para el Desarrollo Sostenible: perspectiva regional y de derechos humanos, Comisión Económica para América Latina y el Caribe (CEPAL), 2018.

JiMENEZ, G. et al., "Overview of systematic reviews of advance care planning: summary of evidence and global lessons", Journal of Pain and Symptom Management, vol. 56, n. ${ }^{\circ} 3,2018,438-459$.

Johnson, S. et al., "Advance care planning: is quality end of life care really that simple?”, Internal Medicine Journal, vol. 47, n. ${ }^{\circ}$ 4, 2016, 390-394.

Jonsen, A., "Dying right in California, The Natural Death Act", Clinical Toxicology, vol. 13 , n. ${ }^{\circ} 4,1978,513-522$.

Kernell-SchiffMAn, I. y Werner, P., "Knowledge regarding advance care planning: a systematic review", Archives of Gerontology and Geriatrics, vol. 73, 2017.

Lathrop, F., "Protección jurídica de los adultos mayores en Chile", Revista Chilena de Derecho, vol. 36, n. ${ }^{\circ}$ 1, 2009, 77-113. 
Leal, M. et al., "Papel del médico de familia en la cumplimentación y registro del documento de instrucciones previas", Semergen - Medicina de Familia, vol. 41, n. ${ }^{\circ} 3,2014,164-167$.

Lum, H. et al., "Advance care planning in the elderly", Medical Clinics of North America, vol. 99, n. ${ }^{\circ}$ 2, 2015, 391-403.

Lum, H. et al., “A group visit initiative improves advance care planning documentation among older adults in primary care", JABFM. Journal of the American Board of Family Medicine:, vol. 30, n. ${ }^{\circ}$, 480-490.

Lupatatzis, B. y Krones, T., “Die Patientenverfügung 'Plus' - das Konzept des Advance Care Planning (ACP)”, Praxis, vol. 106, n. ${ }^{\circ} 25,2017,1369-1375$.

Macfarlane, E., "Dialogue, remedies and positive rights: Carter v. Canada as a Microcosm of past and future issues under the Charter of Rights and Freedom", Ottawa Law Review, vol. 49, n. ${ }^{\circ}$ 1, 2017, 107-129.

Mémeteau, G., "L'affaire Lambert", Médecine \& Droit, vol. 2014, n. 129, 2014, 129-134.

Montalvo, F., "Límites a la autonomía de voluntad e instrucciones previas: un análisis desde el derecho constitucional”, Derecho y Salud, vol. 20, n. ${ }^{\circ}$ 1, 2010, 71-95.

Monteiro, R. y Silva Junior, F., “Advance directive: historical course in Latin America”, Revista Bioética, vol. 27, n. ${ }^{\circ}$ 1, 2019, 86-97.

Nicolas-Vullierme, L. et al. " "De l'opportunité d'une nouvelle loi sur la fin de vie?", Médecine \& Droit, vol. 2018, n. ${ }^{\circ} 152,2018,105-111$.

OsSANDón, M. M., "El derecho a rechazar tratamientos médicos, ¿un reconocimiento del derecho a disponer de la propia vida?", Revista Derecho Público Iberoamericano, . $^{\circ} 2,2013,153-204$.

Pautassi, L., "Del boom del cuidado al ejercicio de derechos", [en línea], Sur. Revista Internacional de Derechos Humanos, vol. 13, n. ${ }^{\circ} 24,2016,35-42$, disponible en: https://sur.conectas.org/wp-content/uploads/2017/02/3-sur-24-esp-laura-pautassi.pdf [consultado el 10 de julio de 2019]. 
Pereira, R., "Human handicap and self-determination: compassion and insensibility in the Vincent Humbert case", História, Ciências, Saúde-Manguinhos, vol. 14, n. ${ }^{\circ} 1,119-134$.

PIERs, R. et al., "Advance care planning in terminally ill and frail older persons", Patient Education and Counseling. Quality of Communication from the Patient Perspective, vol. 90, n. 3, 2013, 323-329.

PIERs, R. et al., "Advance care planning in dementia: recommendations for healthcare professionals", вMC Palliative Care, vol. 17, n. ${ }^{\circ}$ 1, 2018, 1-17.

REY, F., "La discriminación múltiple, una realidad antigua, un concepto nuevo", Revista Española de Derecho Constitucional, n. ${ }^{\circ} 84,2008,251-283$.

Riveros, C. et al., "El maltrato estructural a persona mayores en Chile y la necesidad de formular un índice multidimensional", Revista Universum, vol. 32, n. ${ }^{\circ}$, 2017, 163-176.

Riveros, C. y BARCiA, R., "Obligación ética y jurídica de prevenir y reducir al máximo la aparición de nuevas discapacidades en las personas mayores en Chile", Acta Bioethica, vol. 21, n. ${ }^{\circ}$ 2, 2015, 207-215.

Singer, P. et al., "Bioethics for clinicians: 6. Advance care planning", cmaJ. Canadian Medical Association Journal, vol. 155, n. ${ }^{\circ} 12,1996,1689-1695$.

Sudore, R. et al., "Defining advance care planning for adults: a consensus definition from a multidisciplinary delphi panel", Journal of Pain and Symptom Management, vol. 53, n. ${ }^{\circ}$ 5, 2017, 821-832.

TAYLOR, J., "What are 'best interests'? A critical evaluation of 'best interests' decision-making in clinical practice", Medical Law Review, vol. 24, n. ${ }^{2}$ 2, 2016, 176-205.

Troncoso, D., "Cuidado informal a mayores dependientes en Chile: quiénes cuidan y con qué políticas sociales cuentan”, América Latina Hoy, vol. 71, 2015, 86101.

Toro, R., Conocimientos y actitudes de usuarios, médicos y enfermeras sobre las instrucciones previas en el Área Asistencial Este de la Comunidad de Madrid, [en línea], tesis de doctorado, Universidad de Alcalá,Alcalá de Henares, 2014, disponible en: https://ebuah.uah.es/dspace/bitstream/handle/10017/20563/Tesis\%20 
Rafael\%20Toro\%20Flores.pdf?sequence=1\&isAllowed=y [consultado el 3 de julio de 2019].

VERONESI, P., "Diritto di vivere, libertà di morire? Il caso italiano di Eluana Englaro", en Presno, M. (coord.), Autonomía personal, cuidados paliativos y derecho a la vida, Oviedo, Universidad de Oviedo, 2011, 112-121.

WIESING, U. et al., "A new law on advance directives in Germany”, Journal of Medical Ethics, vol. 36, n. ${ }^{\circ} 12,2010,79-83$.

ZitToun, R., "La sédation continue jusqu'à la mort. Une voie française pour les soins de fin de vie?", La Presse Médicale, vol. 45, n. ${ }^{\circ}$, part 1, 2016, 670-675.

ZúÑIgA, A., "Derechos del paciente y eutanasia en Chile", Revista de Derecho, Valdivia, vol. 21, n. ${ }^{\circ} 2,2008,111-130$.

ZúÑIGA, A., "La nueva ley de derechos del paciente: del modelo de la beneficencia al modelo de autonomía", Revista Médica de Chile, vol. 141, 2013, 123-124. 